\title{
Recent approaches in geodesy and geomatics for structures monitoring
}

\author{
Alessandro Capra $\cdot$ Eleonora Bertacchini • \\ Cristina Castagnetti · Riccardo Rivola • \\ Marco Dubbini
}

Received: 14 April 2015/Accepted: 28 April 2015/Published online: 22 May 2015

(C) The Author(s) 2015. This article is published with open access at Springerlink.com

\begin{abstract}
Monitoring the time history of structures and infrastructures has always been an important area of application of geodetic and geomatic methods. Here we shall concentrate on the item of monitoring ancient buildings because, beyond its intrinsic interest, it provides a good illustration of some of the most recent monitoring techniques. Identifying the overhang, progressive changes of inclination, differential movements of the structure and detailing the study of structural elements are just some examples of the many fundamental and necessary information for structural engineers. Those data are required to study and analyze the behavior of a structure with the purpose to assess the stability. Looking at the several methods offered by Geomatics, laser scanning appears to be the best technology to provide an effective 3D solution to those requirements. Surveying by means of a terrestrial laser scanner, allows to detect a huge number of 3D information with high accuracy in a relatively short time and high accuracy. Just analyzing the point clouds, interesting information along with useful products can be obtained in order to draw some considerations about the investigated structure. This research aims at suggesting a new
\end{abstract}

Peer reviewed version of the paper presented at conference on Geodesy and Geomatics held at Accademia Nazionale dei Lincei in Rome on June 3, 2014.

A. Capra $(\bowtie) \cdot$ E. Bertacchini · C. Castagnetti · R. Rivola DIEF, Department of Engineering Enzo Ferrari, University of Modena and Reggio Emilia, Strada Vignolese 905,

41125 Modena, MO, Italy

e-mail: alessandro.capra@unimore.it

M. Dubbini

DiSCi, Department History Culture Civilization, Headquarters of Geography, University of Bologna, via Guerrazzi 20, 40125 Bologna, Italy philosophy for using 3D models in a diagnostic perspective in order to study structures along with their actual dimensions, their stability and so on. Traditionally, indeed, laser scanning is chosen for artistic and architectural studies and the resulting three-dimensional model represents what often is of concern. Furthermore, the use of more classic techniques, such as total station and digital leveling, and LST is fundamental as an integrated approach for the monitoring of ancient buildings. The integration of different techniques allow a redundancy of observation and the possibility to verify the results obtained form independent techniques as is shown throughout some experimental applications.

Keywords Geodesy · Monitoring · Structures · Integrated systems · Geomatics · 3D Survey · Laser scanning

\section{Introduction}

Traditional instruments and methodologies showed till today high potentiality in obtaining significant results in structure monitoring. One of the most recent innovative approaches should be considered the integration of different techniques in order to obtain a remarkable redundancy in observations and data collection; moreover, it is possible to obtain results from different independent techniques that should allow to verify the significance of the results.

We will show in a practical application to the Church an Torre della Sagra in Carpi (Modena) how are the great advantages of integrated approach in a application where classical leveling has been applied for subsidence effect monitoring on the basement of a middle age tower. On the same tower a control deformation network has been applied with high accurate total station. The comparison of 
results of different techniques, that should be considered independent, allowed to verify a significant differential movement of the structure basement in a specific direction. The results forced to maintain a monitoring study in order to evaluate possible risks for the tower stability.

It is well known that structural monitoring traditionally is performed point-wise; it requires very high accuracy (ranging, according to the different monitoring techniques-not only geomatic, from few tenths to hundredths of millimeter or even microns) on the monitored points, which, on the other hand, are usually quite few and localized only in the crucial areas according to the judgement and experience of the structural expert. Therefore, in this framework, conclusions on the behavior of the whole structure are argued from few points, well selected as representative on the basis of the structural knowledge/experience. In addition, it is well known that, even with the last generation terrestrial laser scanners, such a high accuracy cannot be accessed; but, on the contrary, terrestrial laser scanner can jointly monitor a huge amount of points. Now, the key fact is that the behaviors of such points can be reasonably hypothesized to be not independent; on the contrary, they are strongly correlated (at least a large part of them) by the equazione costitutiva of the material which constitutes the monitored structure. Therefore, even if the accuracy about the behavior of each individual point monitored by terrestrial laser scanner cannot be high, it is just its capability to jointly monitor many points supplying a space-wise correlated information which is of relevant value for structural monitoring; in fact, this space-wise information can replace the point-wise one of the traditional approaches and, in addition, it does not require a previous careful selection of monitoring points, thus simplifying the monitoring design.

In summary, the TLS allows to measure a large amount of points with high dense and detailed distribution in a relatively short range of time. The technique is of low time consuming and great effectiveness; moreover, it permits to answer to many operative requirements. As for example the possibility of high-accuracy surveying of architectonic details very difficult to join, like the elements located on the top of towers.

The methodological approach of TLS is due through the application to Asinelli and Garisenda towers in Bologna. Starting from a points cloud, the analysis of the geometry has been performed in order to extract information useful for evaluating geometrical anomalies and possible structural instability. In particular, the inclination, the overhanging, and his variation in time are interesting geometrical elements to measure in a tower.

The 3D model obtained from TLS survey should be used even as a diagnostic instrument of the structure; in fact the geometry of the system should be analyzed in comparison with a theoretical assessment to define the stress on the structure. This approach has been pursued in Dome of Modena application shown in the paper.

\section{The terrestrial laser scanning as a diagnostic instrument and as a tool for the determination of the actual geometry of structures}

The use of laser scanner technology as a diagnostic tool for structural analysis aims at extracting information and parameters that are the starting point for advanced studies on the static and dynamic behavior of a structure (Bertacchini et al. 2010; Castagnetti et al. 2011). Results are undoubtedly of great help to design and plan interventions of consolidation and safeguarding, necessary as a result of the above analysis. The innovation of the proposed methodology is essentially to change the point of view for analyzing and interpreting the laser scanner data on the basis of the final aim which led the survey. Compared to the traditional approach, the difference mainly lies in the extraction of the results; the final product is not only the three-dimensional surface model for simple documentation, qualitative interpretation, and consultation, but the quantitative and accurate identification of geometric anomalies of the structural elements.

Obviously, design and implementation of surveys as well as data processing are similar to any other laser scanning survey, regardless of what is the a-posteriori analysis performed on the points cloud. It should be underlined that the terrestrial laser scanning cannot be considered rigorously a tool for structural deformation monitoring as it is very difficult to have an exact correspondence between the measurement made and the point actually measured, with the high precision required by the aim. It is substantially impossible to achieve an accuracy in the order of some millimeters, even for relatively small acquisition distances (from 5 to $15 \mathrm{~m}$ ).

The researches presented a new and innovative use of laser scanning technique for cultural heritage applications. The basic idea aims at approaching laser scanner data with a critical point of view in order to analyze the complexity of structures as well as their geometry. The attention moves to constituent elements as well as any evidence of cracking or warping, which are critical for the effective stability of the structure. Three-dimensional point clouds can be valuable sources of information for extracting measurements and parameters which are helpful to foresee the structural behavior, for highlighting whether differential displacements and deformations occur or not and making hypothesis about any additional load that increases imposed stresses.

Many efforts were conducted on the study of structures with a main vertical extension such as ancient towers. The 
analysis of verticality of the Asinelli Tower in Bologna (early XII century, masonry construction with a selenite base resting on alluvial soil subject to subsidence) was initially performed by exporting sections of the model at intervals of $5 \mathrm{~m}$ (Bertacchini et al. 2010). The double symmetry of the structure has allowed us to define the geometric barycenter of each section in $3 \mathrm{D}$ space. The trend of the axis connecting the barycenters obtained shows a tiny buckling in the north-south direction (4 cm max), whereas a $211 \mathrm{~cm}$ displacement was detected from the vertical towards west.

This overhang measure is the planimetric distance between the barycenter of the first section that can be identified above the base ( $10 \mathrm{~m}$ elevation) and the top of the tower not including the turret $(90 \mathrm{~m})$. In order to determine a value that is representative of the actual out-of-plumb line of the structure, it is necessary to extrapolate the barycenter of the ground section by prolonging the barycentric axis in the most appropriate way.

By analyzing the surveys carried out in previous periods and the $3 \mathrm{D}$ point model, 3 parts were identified, differing for wall thickness and construction age. In the hypothesis of a linear relationship between out-of-plumb towards west and elevation, the equations of the linear regression lines best representing the lay-out of the tower parts were determined. Angular coefficient and origin ordinate of the three regression straight lines being known, the inclination angle and the position of the barycenter at ground level can be determined immediately. By prolonging the first segment axis straight line to the ground, a $27-\mathrm{cm}$ increase in overhang was obtained. Therefore, the overall tilt of the structure is $238 \mathrm{~cm}$ to the west (Fig. 1).

The TLS surveying has been made on three middle age towers in Emilia Romagna Region that have similar characteristics and that have prevalent mono-dimensional development. The results obtained for Ghirlandina Tower (Modena) (Blasi et al. 2009; Gaindebbiagi et al. 2009) and Torre della Sagra (Carpi, Modena) are shown in Table 1.

The analyses on the Garisenda Tower in Bologna focused on the selenite coating at the base: the sections generated every $10 \mathrm{~cm}$ show a singular behavior taken from the east side of the structure. On the North, West, and South sides, the sections highlight an homogeneous and constant inclination of the respective walls into the interior of the tower from the bottom upwards; however, the same observation can not be made with respect of the East side, which in addition to having a greater inclination does not even seem to be uniform over the entire length of the facade.

To better investigate this alleged geometric anomaly on the East side of the lower end of the tower, it was decided to calculate the relative distances between the point clouds and a reference plane, using an automatic algorithm of inspection. For each side of the tower, it has been generated a reference plane using the four points at the corners of the selenite coating; each plane is therefore, representative of the global trend of the lower portion of the corresponding side. Each plane was then shifted parallel to itself of $50 \mathrm{~cm}$ towards the interior of the structure so that the distances of the points of the wall from the plane itself had to be all of positive sign. In order to make a meaningful and immediate comparison, the point clouds of the four sides have been colored with the same color scale, ranging from blue to red for distances of between 50 and $70 \mathrm{~cm}$ from each reference plane. The blue color shows the points located along the mid-plane of the wall, while proceeding towards the red coloration are highlighted any phenomena geometrically anomalous compared to that plane (Fig. 2).

The analyses carried out on the Cathedral of Modena provides very interesting examples of further investigations Fig. 3. In this case, the overhang of all sides has been defined by carrying out a detailed analysis of deflections from the verticality with respect to the direction orthogonal to the plane of the wall and also with respect to the plane of the wall itself. Both deflections have been considered and composed in order to obtain the resulting overhanging. This is extremely important for structural engineers in order to assess stresses which lead to open outwards of the structure. These considerations are closely tied to evidences of cracks that only expert eyes are able to detect; such visual inspection is helpful to provide confirmation of the obtained results. The mentioned analysis was performed on external walls by examining key architectural elements such as columns, which are well identifiable on the point cloud and also uniformly distributed along the structure. Columns have been found to be representative of the wall behavior because they develop vertically along the entire height of the structure. The deflection angle is estimated by comparing the vertical direction, which is known by means of the dual-axis compensator of the laser scanner as soon as it has been leveled, to the longitudinal axis of the column, which is extracted by the point cloud itself. Then, the same analysis has been performed on internal columns in order to assess whether there is homogeneous behavior between outside and inside or whether differential movements occur. The latter scenario is very dangerous for the stability of the structure. The results obtained from TLS allowed to define the anomalies on pillars and walls orientation with respect with the verticality (Fig. 4); these geometric anomalies have been used in order to perform the strain-sress analysis; the results are shown in a paper "The structural analysis of the Modena Cathedral (Baraccani S, Silvestri S, Gasperini G, Palermo M, Trombetti T, Silvestri E, Lancellotta R, Capra A)" that have been submitted at a special issue of Journal of Architectural Heritage on SAHC 2014. 
Fig. 1 Images of laser scanner surveying preparation, marker installation. Axis inclination and overhang of Asinelli tower (Bologna) though TLS surveying
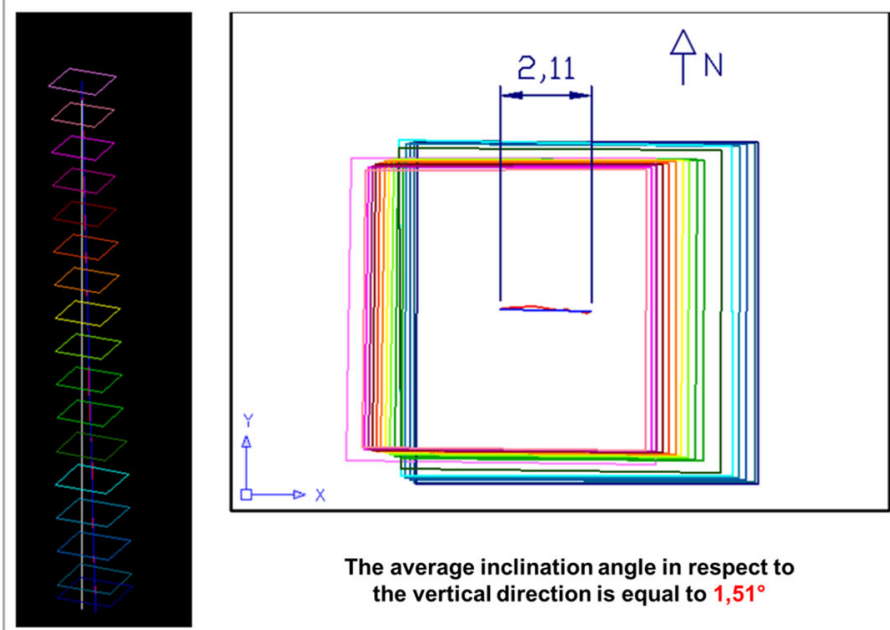

The average inclination angle in respect to the vertical direction is equal to $1,51^{\circ}$
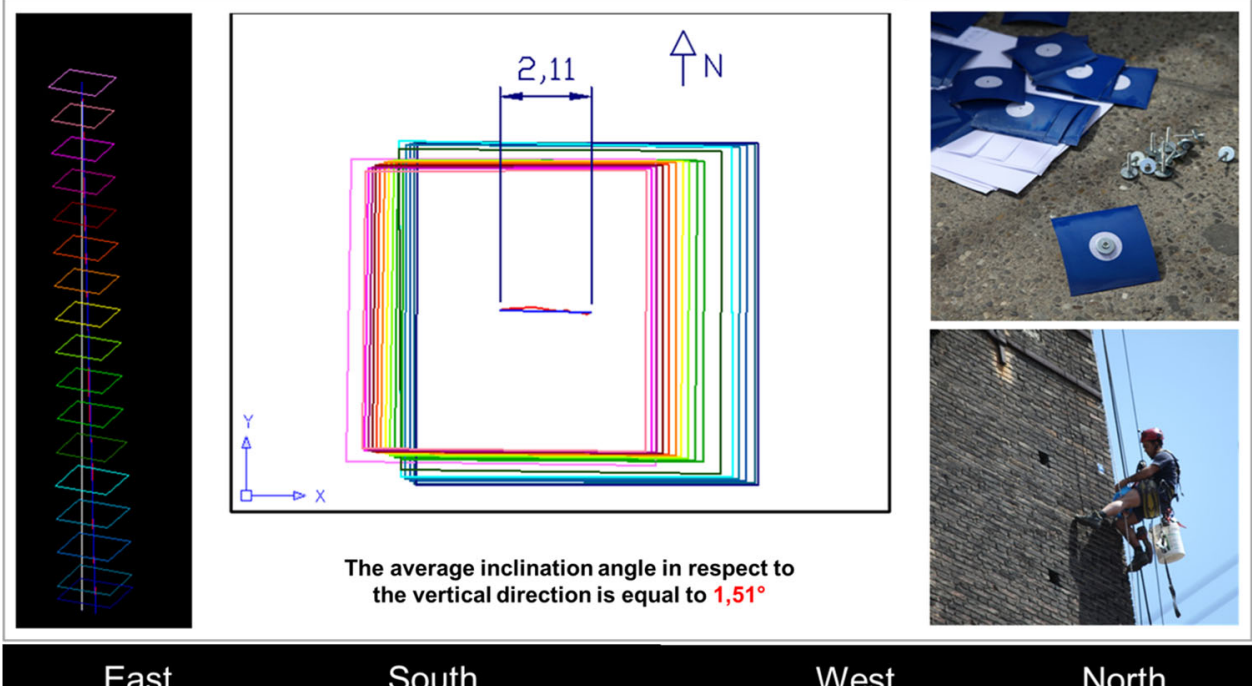

South

West

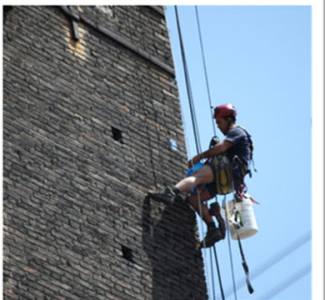

East

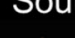

North

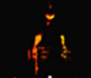

舟:
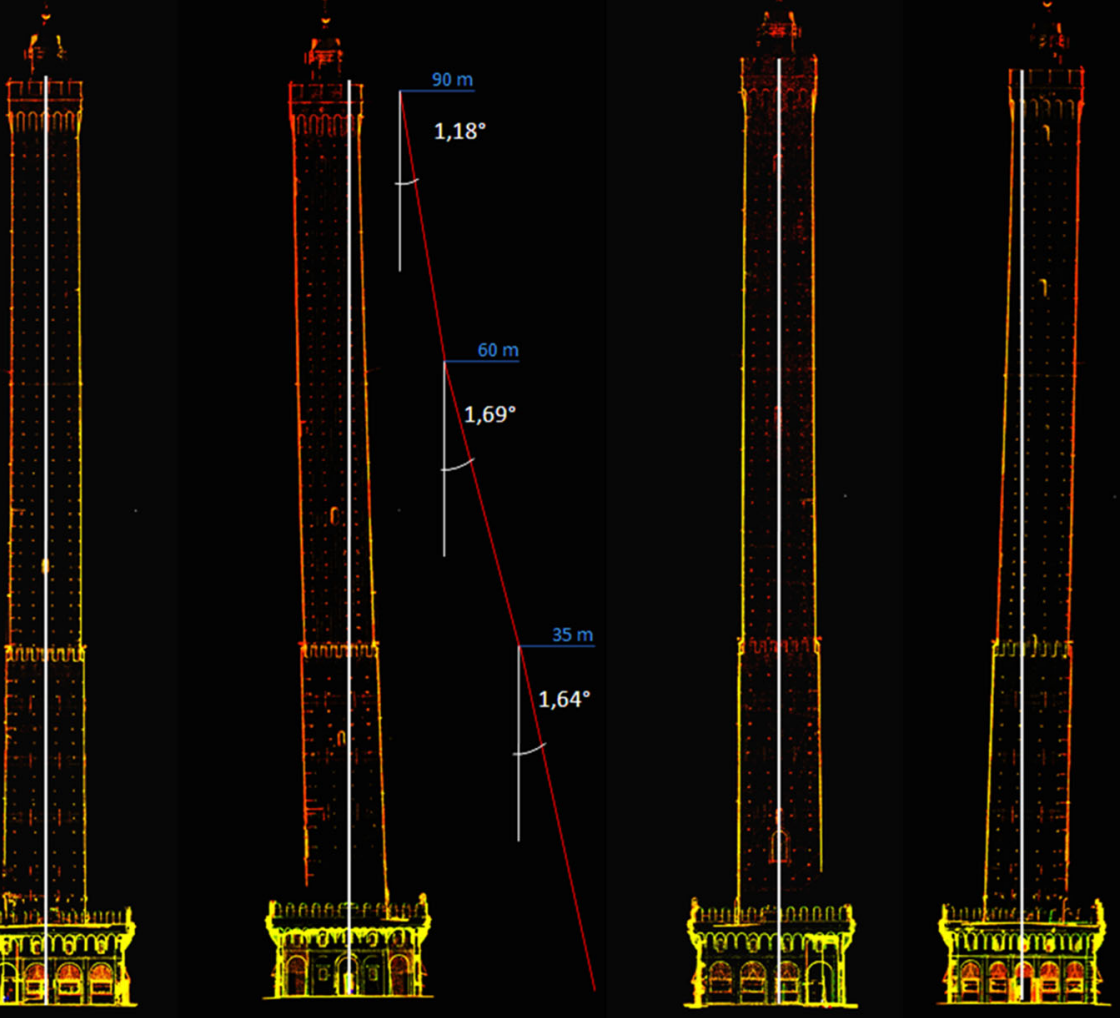

Table 1 Geometry of the middle age towers surveyed with TLS (Bertacchini et al. 2010; Boni et al. 2010)

\begin{tabular}{|c|c|c|c|c|}
\hline & \multirow[t]{2}{*}{ Height (m) } & \multicolumn{2}{|l|}{ Overhang } & \multirow[t]{2}{*}{ Average inclination of barycentric axis } \\
\hline & & Maximum value (m) & Direction & \\
\hline Torre degli asinelli & 97.38 & 2.38 & West & $1.51^{\circ}$ \\
\hline Torre ghirlandina & 88.82 & 1.54 & South-west & $1.04^{\circ}$ \\
\hline Torre della sagra & 48.51 & 0.71 & North & $0.84^{\circ}$ \\
\hline
\end{tabular}



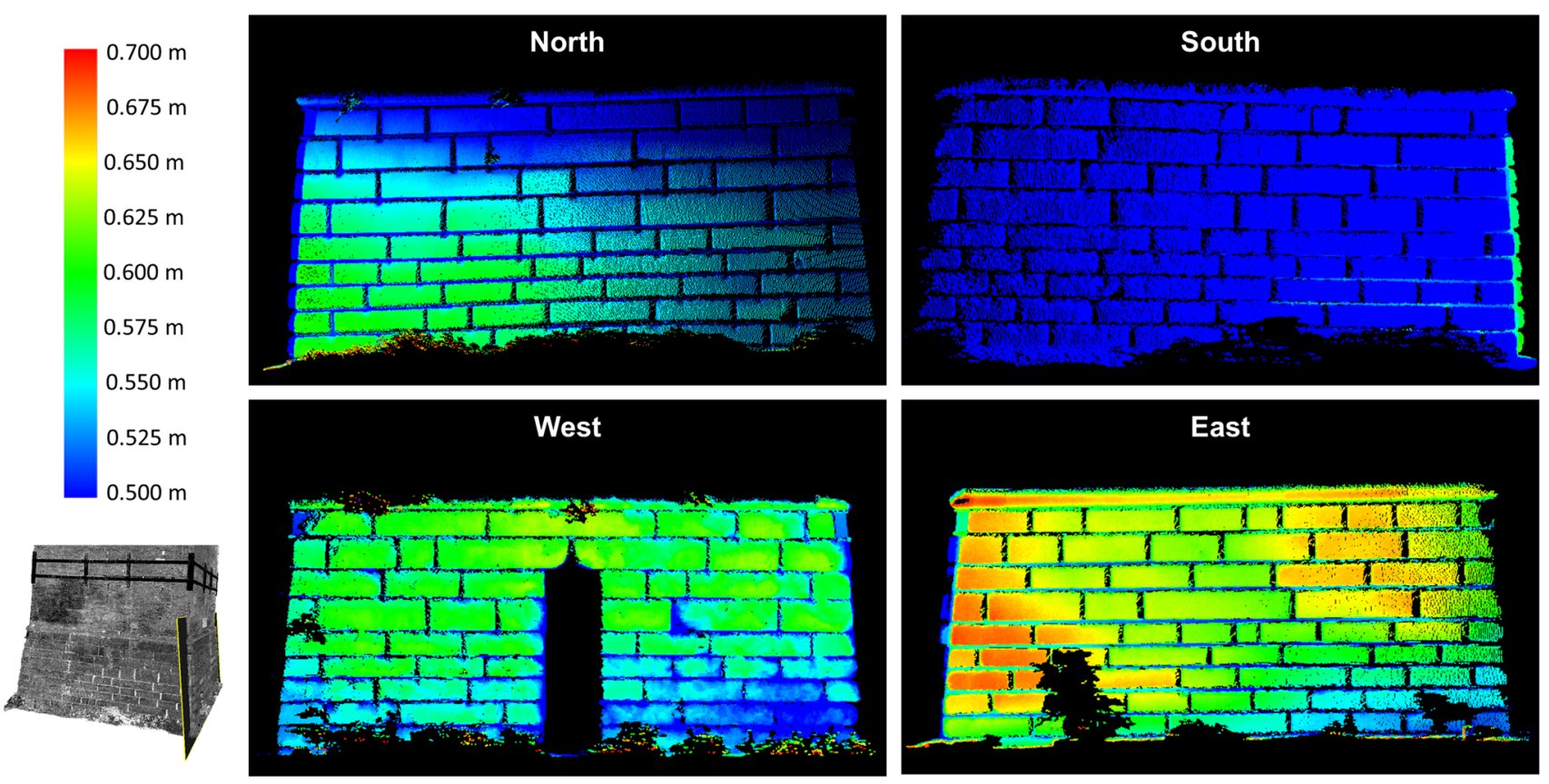

Fig. 2 Surface variation form planarity of Garisenda tower

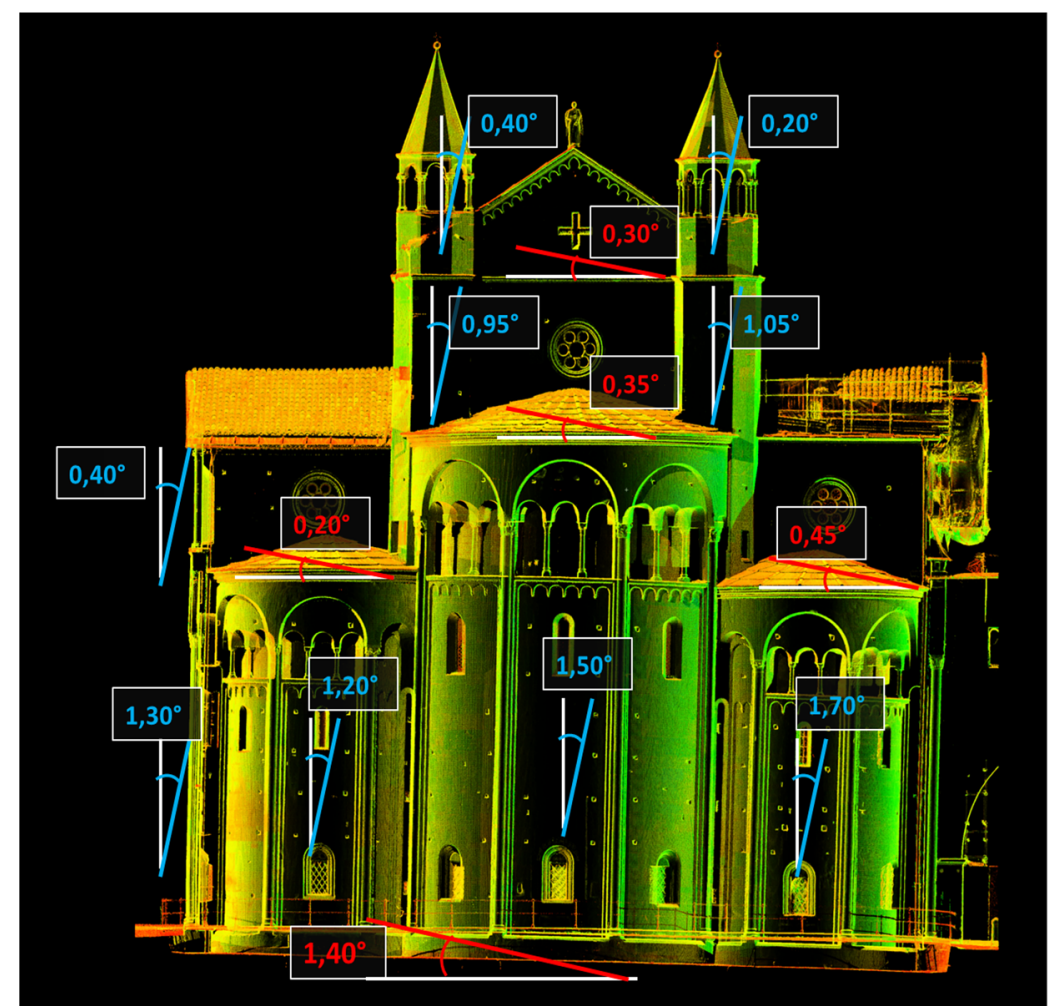

(a)

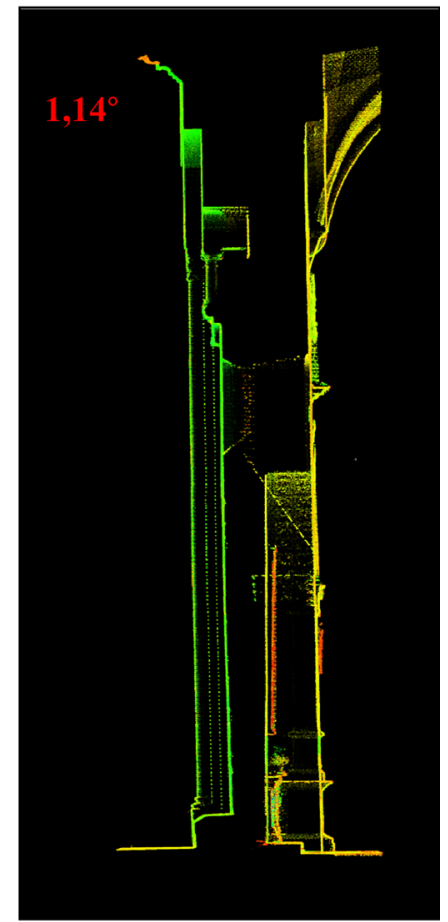

(b)

Fig. 3 a Overhang analysis in south-north direction non Dome absides. b Anomaly with respect to verticality of a section

Further investigations can be performed by analyzing key horizontal elements such as ledges and eaves, which extend longitudinally along the sides of the structure and are distributed at different heights. The verification of the effective horizontality of architectural elements, which should be horizontal, has been carried out on outside 
facades of the Cathedral by means of polylines directly outlined on the point clouds. Having a resolution of about $8 \mathrm{~mm}$, the point cloud allows to accurate outline polylines according to actual geometry of eaves and ledges. By this way the angle between such polyline and the horizontal plane may be computed. To ensure significance to the analysis, the facade has been divided into several parts in order to identify differential movements of portions of the facade (anomaly). Results highlight a differential behavior along the longitudinal extension of the facade. These analysis have also been performed on the apse and led to interesting results in relation to the subsequent steps of construction and restoration. This needs to be validated by comparison with art historians (Figs. 3, 5).

The researches presented confirm the usefulness of laser scanning as a diagnostic tool for structural analysis. The interesting results which have been obtained by analyzing laser scanner point clouds with a diagnostic approach are particularly useful for structural engineers because irregularities and anomalies in the geometry of structures may improve results of numerical simulation models aiming to assess the static vulnerability of ancient artefacts. As a consequence, we may conclude by asserting that laser scanning is a sufficiently complete and accurate technique

Fig. 4 Geometrical anomality with respect to verticality of the pillars
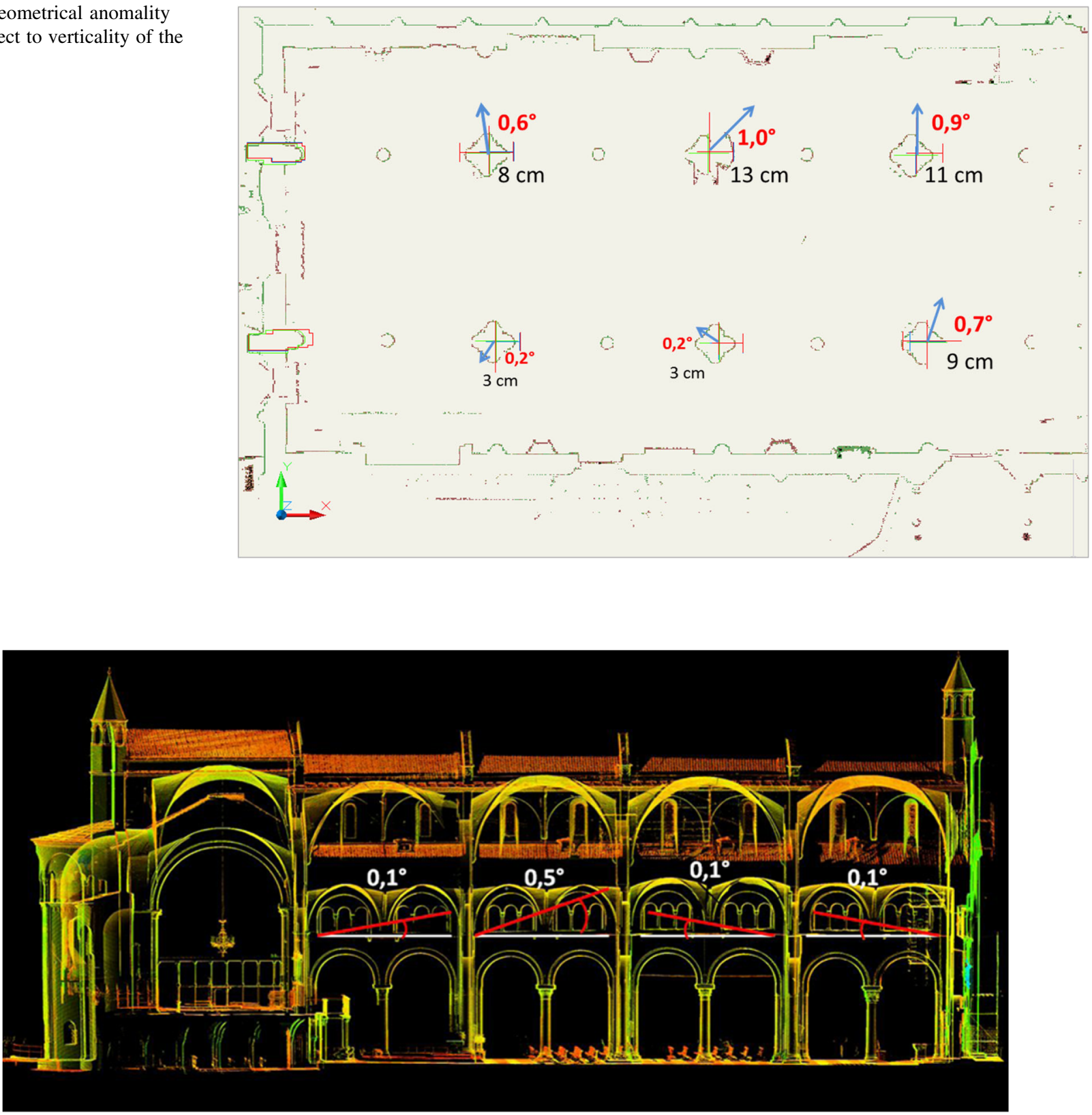

Fig. 5 Analyses of horizontality of apse plan 
to be considered as the basis for geometric investigations. The analysis allowed extracting lot of information about the structure as well as its status. It is worth to underline that it is not possible to interpret results and identified anomalies without integrating and comparing with information about the history of the structure (Castagnetti et al. 2011).

\section{Integrated traditional survey techniques: high precision leveling and total station for the identification of differential movements}

High precision leveling and total station have an essential role in deformation control of structures. It is possible to reach an accuracy at $\mathrm{mm}$ level in the evaluation of deformative field.

It appears particularly interesting the use of the two techniques when the result of measurement should be uncertain if we based conclusions on one method only. If we obtain the same results with the two techniques, that are independent, we should guarantee the goodness of results and successive interpretation and analysis.
Particularly, we obtained an anomalous result in differential vertical movements of some benchmarks measured on Torre della Sagra in Carpi (Modena, Italy). In particular, we had to consider a tragic event of Modena Province earthquake occured in two big seismic events: May and July 2012. The integrated technique application allowed to evaluate the effects of earthquake on Torre della Sagra structure.

In the mean time, the leveling network has been controlled with a 3D network with high accurate total station measurements. Total station surveys and geometric leveling campaigns were performed over 3 years, in the period 2010-2013, twice a year, simultaneously at each time. Some images of the benchmarks and of the total station are shown in Fig. 6e1, e2, e3, and e4.

Therefore, it was possible to proceed in a comparison of the results of each technique, in order to validate the value of a possible deformation of the tower. The comparison of results was significative only after 3 years, considering the entity of the movements and the different degree of precision of each technique, under millimetric for the geometric leveling and millimetric for the 3D adjusted network performed with the total station.
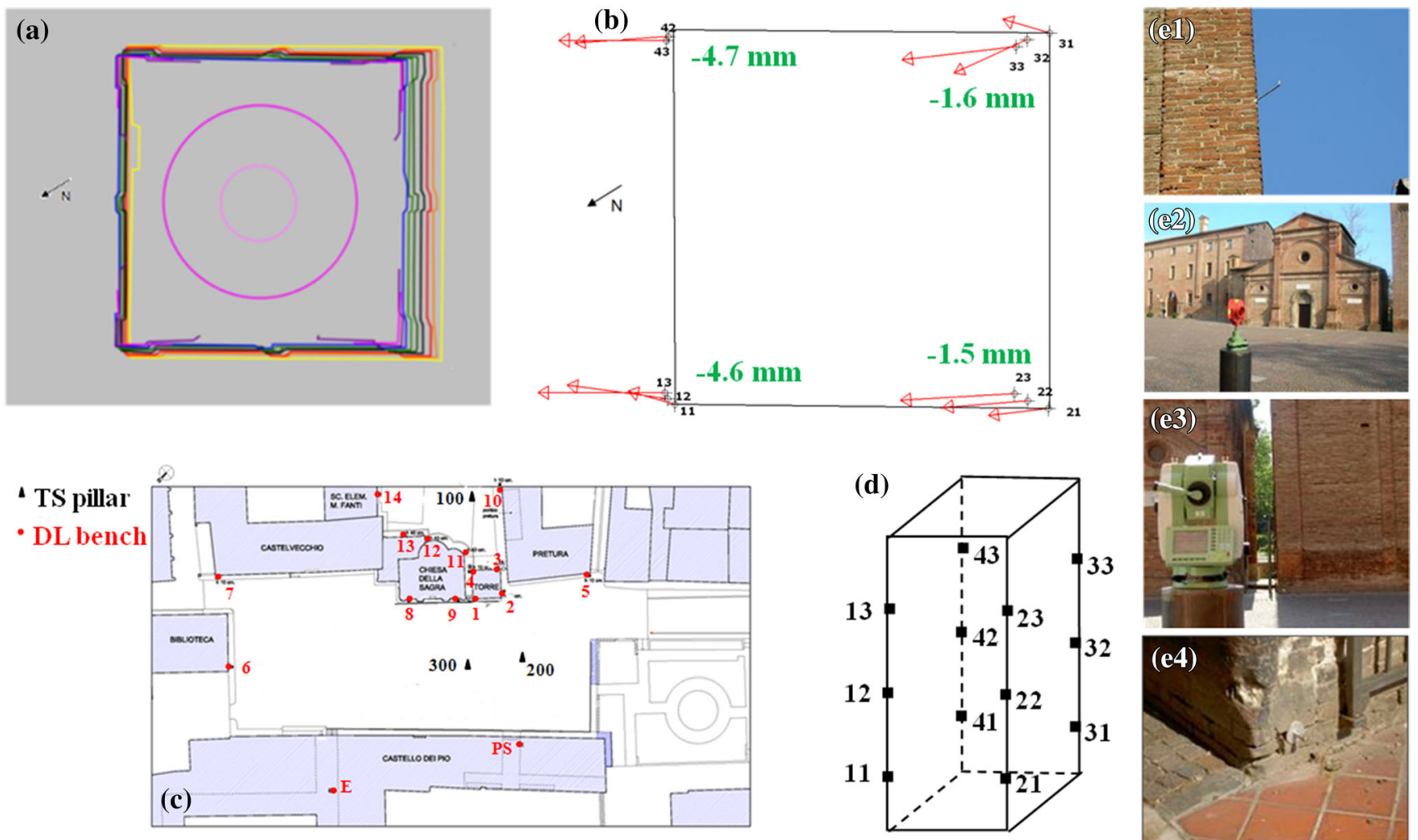

Fig. 6 Torre della Sagra, Carpi (Modena, Italy). a Sections of the tower at different heights, derived from the LS 3D model. Top View (pink, circle); bottom view (yellow, quite square poligon). b 2D displacement vectors from TS and vertical displacement values from DL. c Investigate area and benchmarks (1-14, E, PS) positions for DL and TS pillars $(100,200,300)$. d Assonometric view and position of TS benchmarks on the tower. E1 Image of the TS benchmark on the tower. E2 Image of the TS prism on the pillar. E3 Image of the TS on the pillar. E4 Image of a vertical benchmark of the DL on the tower (color figure online) 
The leveling network (Fig. 6c) was performed referring the origin of heights to local benchmark PS; the connection of the local leveling network with "external" benchmarks has been realized with the connection to Leveling Network of the Municipality of Carpi, particularly with two points located, respectively, at about $150 \mathrm{~m}$ (benchmark 108_01) and about $350 \mathrm{~m}$ (benchmark 108_02) from PS. In height adjustment and computation, PS was assumed fixed over time for step 1 and 2 and the benchmarks 108_01 e 108_02 were assumed fixed and frozen at the 2007 year of their last survey by the municipality.

For the determination of vertical displacements, the precision reached was at $2 \sigma$ of $0.1 \div 0.2 \mathrm{~mm}$, therefore, only relative differences larger than $0.2 \mathrm{~mm}$ were considered as significative.

The 3D control network, measured with total station, a survey was performed with a total station on the pillars $(100,200,300)$ at each campaign, following standard rules and procedures properly defined to reduce the errors. The pillar position was found fixed, considering that the variation of the coordinates was within the precision of the network adjustment (at $2 \sigma$ variation $<3 \mathrm{~mm}$ ). The network adjustment was performed on the benchmarks installed on the tower (Fig. 6d), considering as fixed points the positions of the instrument station $(100,200,300)$ and assuming as invariant the coordinates computed on a local reference frame in the first campaign.

For each survey the air temperature, pressure, and relative humidity were also collected, therefore, atmospheric corrections were performed (Bertacchini et al. 2011; Castagnetti et al. 2013) on the slope distance.

Comparing the precision reached on each adjustment over time, there were only little variations, thus it was not necessary to perform the error propagation for the determination of the precision of computed displacement: the precision used was the lower one, even if very little differences occurred.

Figure $6 \mathrm{~b}$ shows $2 \mathrm{D}$ vectors representing the movement direction and entity detected by the TS, with a factor of magnification of 10 points, to make them more readable; the geometric leveling (DL) confirms, for the benchmark of the tower, the vertical displacements found with the total station (numerical value near the edges).

The total station and the geometric leveling are independent techniques, thus the integrated use of different instruments and methods led to the same results, which are then validated. The two techniques confirmed the remarkable effects of 2012 earthquake. It would be necessary to perform further campaigns to confirm the result over time in order to distinguish the effect of an extraordinary event, like earthquake, and the effect of subsidence displaying a long-period behavior. In 3 years there we can only collect the first significant results with both techniques:
- all monitoring points are lowering with respect to PS, which is the reference point for the vertical network;

- PS is lowering with respect to the points outside the local network, this should be considered in a more extended reference frame, to appreciate the total lowering and not only the differential ones which are those important for the risk analysis for the structure.

- there are differential effects on the different floor of the tower and a torsional effect is quite evident. Figure $6 a$, realized taking sections at different heights of the 3D model of the tower realized by means of a laser scanner survey, shows the asset of the tower, which hangs in the North direction, to the adjacent church (Table 1).

\section{Conclusion}

The field of geodetic and geomatic applications to structural monitoring the attention has been focused on relatively recent approaches such as the terrestrial laser scanner and the intensive use of integrated techniques.

The described research proposes a new and innovative use of laser scanning technique for cultural heritage applications: attention is not paid to artistic and architectonic elements and the aim is not only to return the three-dimensional surface model of the investigated structure for qualitative assessments (traditional approach). The basic idea aims at approaching laser scanner data with a critical point of view in order to analyze the complexity of structures as well as their geometry. The attention moves to constituent elements as well as any evidence of cracking or warping, which are critical for the effective stability of the structure. Three-dimensional point clouds can be valuable sources of information for extracting measurements and parameters which are helpful to foresee the structural behavior, highlighting whether differential displacements and deformations occur or not and making hypothesis about any additional load that increases imposed stresses. These results are fundamental to plan interventions for strengthening and securing the structure. This confirms the usefulness of laser scanning as a diagnostic tool for structural analysis. As a consequence, the research may conclude that laser scanning is a sufficiently complete and accurate technique to be considered as the basis for geometric investigations. The above discussion confirms that the analysis allowed to extract lot of information about the structure as well as its status. It is worth to underline that it is not possible to interpret results and identified anomalies without integrating and comparing with information about the history of the structure. The research is a work in progress and further steps will focus on multidisciplinary 
approach in order to provide a comprehensive methodology for studying cultural heritage.

The utilization of different instruments and methodology allowed to obtain important advantages in deformation control of structures. In fact it is possible to exploit the potentiality of different instruments and to reach a relevant redundancy of observations. The most important result obtainable in the integrated approach is the possibility of verifying the final result using different and independent techniques that can guarantee the quality of deformation filed interpretation.

Open Access This article is distributed under the terms of the Creative Commons Attribution 4.0 International License (http:// creativecommons.org/licenses/by/4.0/), which permits unrestricted use, distribution, and reproduction in any medium, provided you give appropriate credit to the original author(s) and the source, provide a link to the Creative Commons license, and indicate if changes were made.

\section{References}

Bertacchini E, Boni E, Capra A, Castagnetti C, Dubbini M (2010) Terrestrial laser scanner for surveying and monitoring middle age towers. In: Atti XXIV FIG International Congress, Aprile, Sydney, pp 11-16

Bertacchini E, Capra A, Castagnetti C, Corsini A (2011) Atmospheric corrections for topographic monitoring systems in landslides. In: XXV FIG working week 2011. Marrakesh, Marocco, 18-22 maggio 2011, vol. TS06A n. 4905, p 1-16, Copenaghen: FIG, ISBN: 9788790907921

Blasi C, Coisson E, Capra A, Lancellotta R (2009) I dati del monitoraggio per la comprensione dei movimenti della Torre Ghirlandina: un progetto per la conservazione. Luca Sossella Editore, Roma, Aprile 2009. ISBN 9788889829721

Boni E, Capra A, Bertacchini E, Castagnetti C (2010) Terrestrial laser scanner for surveying and monitoring middle age tower. In: Proceedings of XXIV FIG International Congress 2010. ISBN 978-87-90907-87-7

Castagnetti C, Bertacchini E, Capra A, Dubbini M (2011) Il laser scanning terrestre per l'analisi di edifici di interesse storico ed artistico. Geomatica: le radici del futuro-Tributo a Sergio Dequal e Riccardo Galetto, 2011, pp 99-107. ISBN 88-9019396-4

Castagnetti C, Bertacchini E, Corsini A, Capra A (2013) Multisensors integrated system for landslide monitoring: critical issues in system setup and data management. Eur J Remote Sensi 46:104-124. doi:10.5721/EuJRS20134607

Giandebiaggi P, Zerbi A, Capra A (2009) Il rilevamento della Torre Ghirlandina (The surveying of Ghirlandina Tower). La Torre Ghirlandina: un progetto per la conservazione. Luca Sossella Editore, Roma, Aprile 2009. ISBN: 9788889829721 